\title{
Modeling and Control of Piezoelectric Actuators for Active Physiological Tremor Compensation
}

\author{
U-Xuan Tan ${ }^{1}$, Win Tun Latt ${ }^{1}$, Cheng Yap Shee ${ }^{1}$, Cameron Riviere ${ }^{2}$ \\ and Wei Tech Ang 1 \\ ${ }^{1}$ Nanyang Technological University ${ }^{2}$ Carnegie Mellon University \\ ${ }^{1}$ Singapore, ${ }^{2}$ United States
}

\section{Introduction}

Humans have intrinsic limitations in manual positioning accuracy due to small involuntary movements that are inherent in normal hand motion. Among the several types of erroneous hand movements, physiological tremor is well studied and documented. Physiological tremor is roughly sinusoidal, in the frequency band of $8-12 \mathrm{~Hz}$, and measures about $50 \mu \mathrm{m}$ rms or more in each principal direction. Physiological hand tremor degrades the quality of many micromanipulation tasks and is intolerable in certain critical applications such as microsurgery and cell manipulation. In the human hand, humans are already in possession of a high dexterity manipulator with an unbeatable user interface. Hence, instead of replacing the human hand with a robotic manipulator, Riviere et al. [Riviere et al., 2003] proposed a completely handheld ophthalmic microsurgical instrument, named Micron, that senses its own movement, distinguishes between desired and undesired motion, and deflects its tip to perform active compensation of the undesired component (Fig. 1).

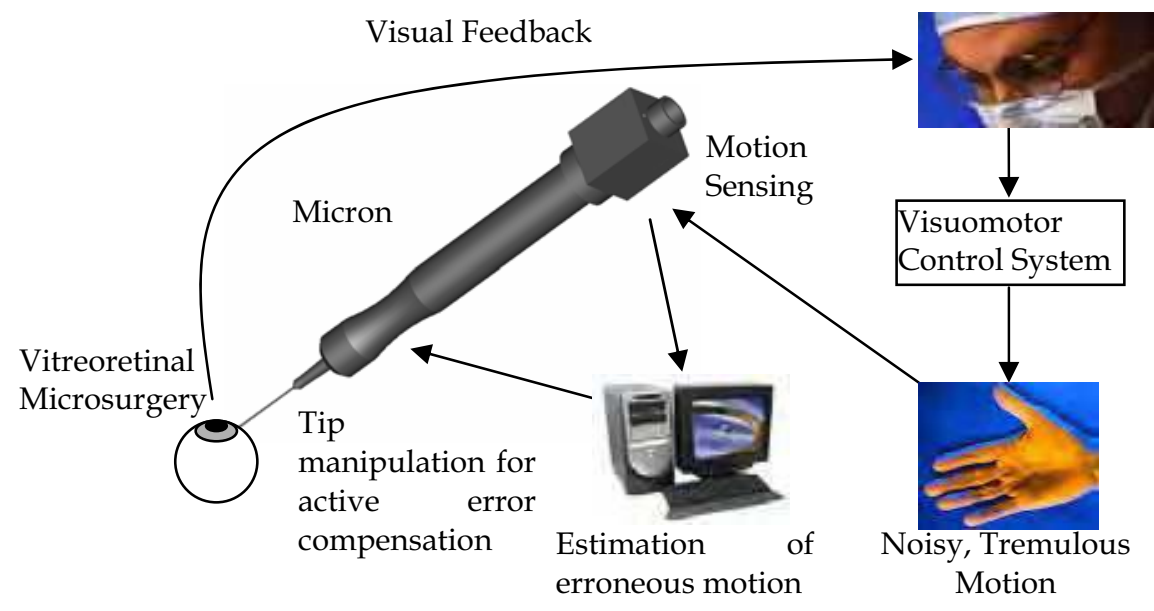

Figure 1. Overview of Micron

Source: Human-Robot Interaction, Book edited by Nilanjan Sarkar,

ISBN 978-3-902613-13-4, pp.522, September 2007, Itech Education and Publishing, Vienna, Austria 
This active compensation approach presents several technical challenges in the control of the robotic mechanism that manipulates the intraocular shaft. The accuracy required can go down to a few microns for applications like microsurgery. To achieve that, the controller must be able to perform tracking control of the actuator to sub micron level. Physiological tremor is typically $8-12 \mathrm{~Hz}$. Controlling the actuator to accurately track a motion of about $10 \mathrm{~Hz}$ is beyond the system bandwidth of many actuators. In order to actively compensate the tremor motion, real-time issue is another concern. Minimal phase difference is permitted as phase difference will result in larger tracking error. Most controllers, which introduce phase difference, are therefore not recommended. Thus, an open loop feedforward controller is proposed. To make things even more challenging, tremor is not rateindependent. The tremor frequency of a person modulates with type of motion and time.

Due to the high velocity and good resolution required, actuators involving smart materials like piezoelectric are proposed. However, their hysteretic behavior makes control difficult. In this chapter, the authors used the Prandtl-Ishlinskii (PI) hysteresis model to model the hysteretic behavior. The PI hysteresis model is a simple model. Its inverse can be obtained analytically, shortening the computational time and making it ideal for real-time application. Since the PI operator inherits the symmetry property of the backlash operator, a saturation operator is used to make it not symmetrical. The inverse model, also of the PI type, is used as the feedforward controller. A slight modification is also proposed to account for the one-sided characteristic of the actuator.

To accommodate human tremor's modulating frequency behavior, a rate-dependent hysteresis model is proposed. As the velocity or load increases, the slope of the hysteretic curve at the turning point tends to 0 and then negative, creating a singularity problem. This chapter also shows how the problem can be overcome by mapping the hysteresis through a transformation onto a singularity-free domain where the inversion can be obtained.

\section{Piezoelectric Actuators}

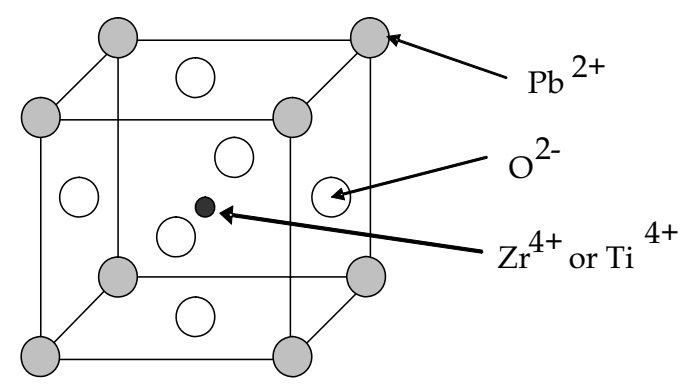

Figure 2. A Crystal Unit Cell of PZT Ceramics

Piezoelectric ceramic has been of increasing interest due to the developments in precision engineering and micro-positioning applications, especially in situations wherein precision, high frequency, and compactness are needed. Piezoelectric ceramic is also playing an increasing role in the medical industry as it is compatible with sensitive medical devices like MRI. Choi et al. [Choi et al., 2005] used piezoelectric actuators for their microsurgical instrument. One common example of piezoelectric ceramic is PZT ceramic. PZT is a solid 
solution of $\mathrm{PbZrO}_{3}$ and $\mathrm{PbTiO}_{3}$ and the general formula is $\mathrm{Pb}\left(\mathrm{Zr}_{\mathrm{y}} \mathrm{Ti}_{1-\mathrm{y}}\right) \mathrm{O}_{3}$. $\mathrm{PZT}$ has the pervoskite $\mathrm{ABO}_{3}$ structure (Fig. 2).

When a voltage is applied across the ceramic, the potential difference causes the atom at the centre ( $\mathrm{Zr}$ or $\mathrm{Ti}$ ) to displace (Fig. 3). A pole is thus induced and the net polarization in the PZT ceramic changes. This results in the deformation of the material. An opposite phenomenon occurs when the ceramic is loaded with a force. A change in polarization occurs and a voltage potential difference is induced. This explains why piezoelectric materials are commonly used both as actuators and sensors.

Figure 3. Polarization

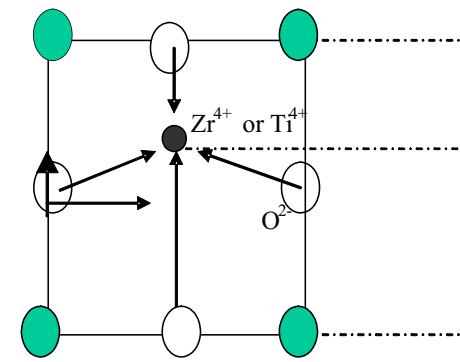

A piezoelectric ceramic is an excellent choice because of its ability to output a large force, large operating bandwidth and fast response time. Unfortunately, effective employment of piezoelectric actuators in micro-scale dynamic trajectory-tracking applications is limited by two factors: (1) the intrinsic hysteretic behavior of piezoelectric material, and (2) structural vibration. The maximum hysteretic error is typically about $15 \%$. To make matters worse, the hysteresis path changes according to rate (Fig. 4), as time is needed for the atoms to move and switching of the polarization to adjust and settle down. Landauer et al. [Landauer et al., 1956] discussed about the dependence of the polarization, in barium titanate, on the rate at which the field is cycled.

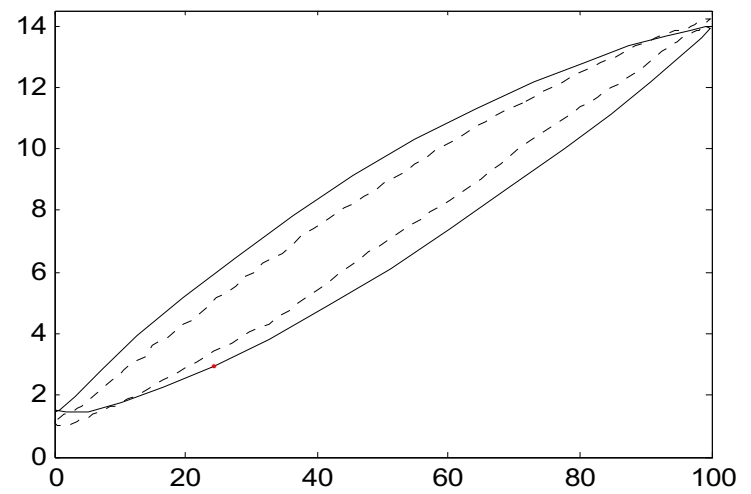

Figure 4. Hysteresis Path at different frequency

While research on rate-independent control of piezoelectric actuators has been extensive, there have been few attempts and little success at controlling the actuator at varying frequency. Hysteresis modeling or compensation can be generally classified into 5 
categories: (1) Linear control with feedforward inverse hysteresis model; (2) Microscopic theories; (3) Electric Charge Control; (4) Phase Control; and (5) Closed-loop displacement control. The more recent methods comprise a hybrid of the methods.

Category (1) relates the underlying understanding of the material at microscopic level with respect to displacement. Landauer et al., 1956 discussed the dependence of the polarization, in barium titanate, on the rate at which the field is cycled. Category (2) makes use of the knowledge that the hysteresis of the actuator's displacement to the applied voltage is about $15 \%$ while the displacement to induced charge is $2 \%$. This motivated Furutani et al. [Furutani et al., 1998] to combine induced charge feedback with inverse transfer function compensation. Category (3) includes Cruz-Hernandez \& Hayward [Cruz-Hernandez \& Hayward, 1998; 2001] proposing the idea of considering phase as a control approach to design a compensator to reduce hysteresis. Category (4) consists of many different approaches. Some proposed incorporating inverse hysteresis model with a controller while others proposed advance controllers like neural network [Hwang et al. 2003], fuzzy logic [Stepanenko et al. 1998], sliding mode [Abidi et al. 2004] and $\mathrm{H}_{\infty}$ control [Chen et al. 1999].

Category (5), a phenomenological approach, is about obtaining a mathematical representation of the hysteresis motion through observation. Phenomenological approach is more commonly used because the underlying physics of the relationship of the smart materials like piezo-actuator's hysteresis path with rate and load is not well understood. Thus, there are many different attempts to derive different mathematical models that best describe the complex hysteretic motion. The inverse model is then used as a feedforward controller to linearize the hysteresis response as shown in Fig. 5.

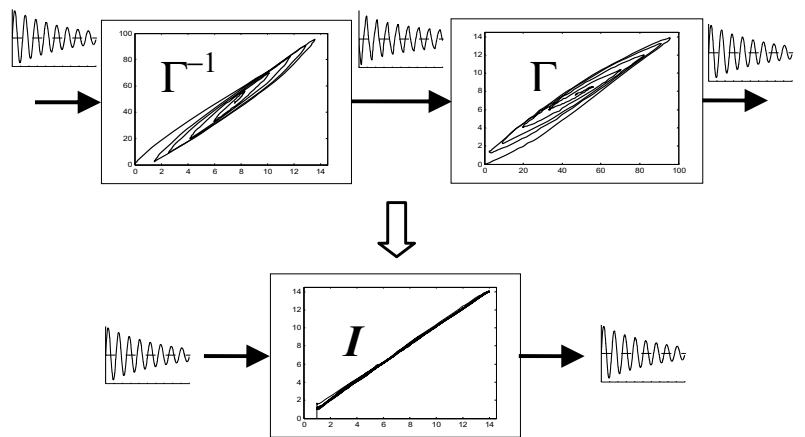

Figure 5. Linearization of Hysteresis Model using Inverse Feedforward Controller

A number of hysteresis mathematical models have been proposed over the years. Hu et al. [Hu et al., 2002] and Hughes et al. [Hughes et al., 1995] proposed using the Preisach model while Goldfarb et al. [Goldfarb et al., 1996; 1997] and Choi et al. [Choi et al., 1997] used Maxwell's model. Tao [Tao, 1995] used the hysteron model. The more recent papers are a variation from the classical models to avoid certain conditions.

Another model is the Prandtl-Ishlinskii model. [Kuhnen \& Janocha, 2001; 2002] and [Janocha \& Kuhnen, 2000] demonstrated that the classical Prandtl-Ishlinskii operator is less complex and its inverse can be computed analytically. Thus, it is more suitable for real-time applications because minimal mathematical computation time is required. Unfortunately, to use the model, the operating frequency must not be too high as the hysteresis non-linearity becomes more severe. Like most models, the classical Prandtl-Ishlinskii model is unable to 
function as a feedforward controller when the largest displacement does not occur at the highest input signal (Fig. 6) as singularity occurs in the inverse.

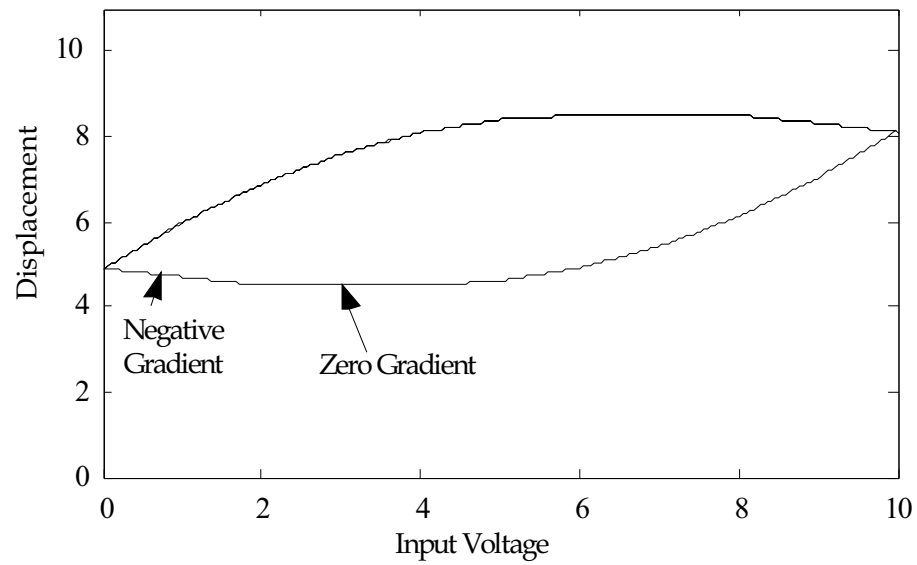

Figure 6. Ill-Conditioned Hysteresis

In this chapter, there are two main contributions: (1) In order to accommodate human tremor's modulating frequency behaviour, a rate-dependent feedforward controller is proposed; and (2) a solution to the inverse of the ill-conditioned hysteresis because as the velocity or load increases, the slope of the hysteretic curve at the turning point tends to 0 and then negative, creating a singularity problem. This is achieved by mapping the hysteresis through a transformation onto a singularity-free domain where the inversion can be obtained.

\section{Hysteresis Modeling}

\subsection{Prandtl-Ishlinskii (PI) Operator}

The elementary operator in the PI hysteresis model is a rate-independent backlash operator. It is commonly used in the modeling of backlash between gears with one degree of freedom. A backlash operator is defined by

$$
y(t)=H_{r}\left[x, y_{0}\right](t)=\max \{x(t)-r, \min \{x(t)+r, y(t-T)\}\}
$$

where $x$ is the control input, $y$ is the actuator response, $r$ is the control input threshold value or the magnitude of the backlash, and $T$ is the sampling period. The initial condition of (1) is normally initialised as

$$
\left.y(0)=\max \left\{x(0)-r, \min \left\{x(0)+r, y_{0}\right)\right\}\right\}
$$

where $y_{0} \in \mathfrak{R}$, and is usually but not necessarily initialized to 0 . Multiplying the backlash operator $H_{r}$ by a weight value $w_{h}$, the generalized backlash operator is

$$
y(t)=w_{h} H_{r}\left[x, y_{0}\right](t) .
$$


The weight $w_{h}$ defines the gain of the backlash operator $\left(w_{h}=y / x\right.$, hence $w_{h}=1$ represents a $45^{\circ}$ slope) and may be viewed as the gear ratio in an analogy of mechanical play between gears, as shown in Fig.7.

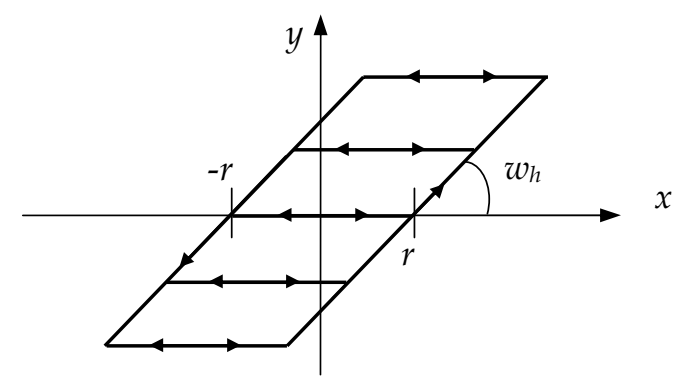

Figure 7. The rate-independent generalized backlash operator is characterized by the threshold or backlash magnitude, $r$, and the weight or backlash operator gain, $w_{h}$

Complex hysteretic nonlinearity can be modeled by a linearly weighted superposition of many backlash operators with different threshold and weight values,

$$
y(t)=\vec{w}_{h}^{T} \vec{H}_{r}\left[x, \vec{y}_{0}\right](t),
$$

with weight vector $\vec{w}_{h}^{T}=\left[w_{h 0} \ldots w_{h n}\right]$ and $\vec{H}_{r}\left[x, \vec{y}_{0}\right](t)=\left[H_{r 0}\left[x, y_{00}\right](t) \ldots H_{r n}\left[x, y_{0 n}\right](t)\right]^{T}$ with the threshold vector $\vec{r}=\left[r_{0} \ldots r_{n}\right]^{T}$ where $0=r_{0}<\ldots<r_{n}$, and the initial state vector $\vec{y}_{0}=\left[y_{00}\right.$ $\left.\ldots y_{0 n}\right]^{T}$. The control input threshold values $\vec{r}$ are usually, but not necessarily, chosen to be equal intervals. If the hysteretic actuator starts in its de-energized state, then $\vec{y}_{0}=\overrightarrow{0}_{n \times 1}$.

Equation (4) is the PI hysteresis operator in its threshold discrete form. The hysteresis model formed by the PI operator is characterized by the initial loading curve (Fig. 8). It is a special branch traversed by equation (4) when driven by a monotonically increasing control input with its state initialized to zero (i.e. $y(0)=0$ ). The initial loading curve is defined by the weight values $\vec{w}_{h}$ and threshold values $\vec{r}$,

$$
\varphi(r)=\sum_{j=0}^{i} w_{h j}\left(r-r_{j}\right), r_{i} \leq r<r_{i+1}, i=0, \ldots, n .
$$

The slope of the piecewise linear curve at interval $i$ is defined by $W_{h i}$, the sum of the weights up to $i$,

$$
W_{h i}=\frac{d}{d r} \varphi(r)=\sum_{j=0}^{i} w_{h j}
$$

The subsequent trajectory of the PI operator beyond the initial loading curve with nonnegative control input is shown as the dotted loop in Fig. 8. The hysteresis loop formed by the PI operator does not return to zero with the control input. This behaviour of the PI operator closely resembles the hysteresis of a piezoelectric actuator.

The backlash operators cause each of the piecewise linear segments to have a threshold width of $2 r$ beyond the initial loading curve. As such, there is no need to define any 
backlash operators beyond the midpoint of the control input range, i.e. $r_{n} \leq 1 / 2 \max$ \{control input\} [Ang 2003]. This also implies that the backlash operators have descending importance from the first to the last, since the first operator is always used and the subsequent operators are only used when the control inputs go beyond their respective threshold values, $r_{i}^{\prime} \mathrm{s}$. Moreover, observations from the piezoelectric hysteretic curves suggest that more drastic changes in the slope occur after the turning points, i.e. in the region of the first few backlash operators. To strike a balance between model accuracy and complexity, the authors propose to importance-sample the threshold intervals $\vec{r}$, i.e., to have finer intervals for the first few backlash operators and increasing intervals for the subsequent ones.

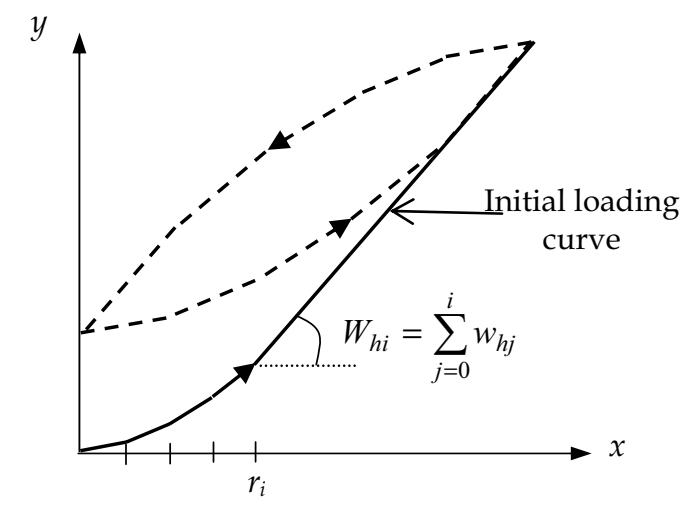

Figure 8 . The PI hysteresis model with $n=4$. The hysteresis model is characterized by the initial loading curve. The piecewise linear curve is defined by the equally spaced threshold values $\vec{r}$ and the sum of the weight values $\vec{w}_{h}$.

\subsection{Modified Prandtl-Ishlinskii (PI) Operator}

The PI operator inherits the symmetry property of the backlash operator about the center point of the loop formed by the operator. The fact that most real actuator hysteretic loops are not symmetric weakens the model accuracy of the PI operator. To overcome this overly restrictive property, a saturation operator is combined in series with the hysteresis operator. The general idea is to bend the hysteresis. A saturation operator is a weighted linear superposition of linear-stop or one-sided dead-zone operators. A dead-zone operator is a non-convex, asymmetrical, memory-free nonlinear operator (Fig. 9). A one-sided dead-zone operator and a saturation operator are given by

$$
\begin{gathered}
S_{d}[y](t)= \begin{cases}\max \{y(t)-d, 0\}, & d>0 \\
y(t), & d=0\end{cases} \\
z(t)=\vec{w}_{s}^{T} \vec{S}_{d}[y](t),
\end{gathered}
$$

where $y$ is the output of the hysteresis operator, $z$ is the actuator response, $\vec{w}_{s}^{T}=\left[w_{s 0} \ldots w_{s m}\right]$ is the weight vector, $\vec{S}_{d}[y](t)=\left[S_{d 0}[y](t) \ldots S_{d m}[y](t)\right]^{T}$ with the threshold vector $\vec{d}=\left(d_{0} \ldots\right.$ 
$\left.d_{m}\right)^{T}$ where $0=d_{0}<d_{1}<\ldots<d_{m}$. For convenience, intervals of $\vec{d}$ between $d_{0}$ and $d_{m}$ need not be equal. Good selection of $\vec{d}$ depends on the shape of the hysteresis loop, and typically involves some trials and errors.

The modified PI operator is thus

$$
z(t)=\Gamma[x](t)=\vec{w}_{s}^{T} \vec{S}_{d}\left|\vec{w}_{h}^{T} \vec{H}_{r}\left[x, \vec{y}_{0}\right]\right|_{(t)} .
$$

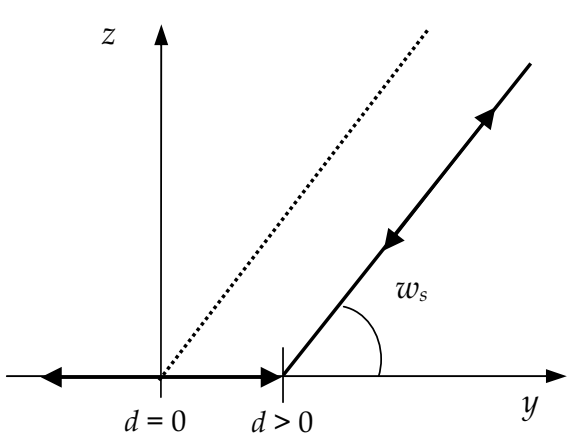

(a)

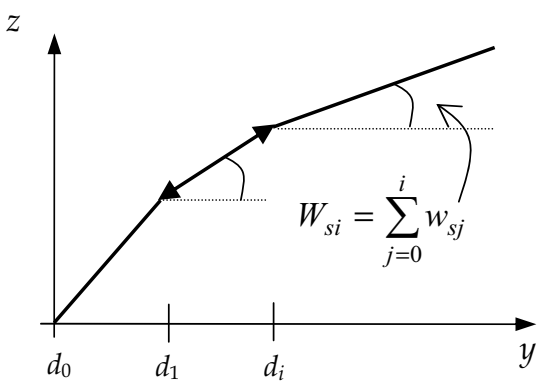

(b)

Figure 9. (a) The one-sided dead-zone operator is characterized by the threshold, $d$, and the gain, $w_{s}$. (b) The saturation operator with $m=2$. The slope of the piecewise linear curve at interval $i, W_{s i}$ is defined by the sum of the weights up to $i$.

\subsection{Parameter Identification}

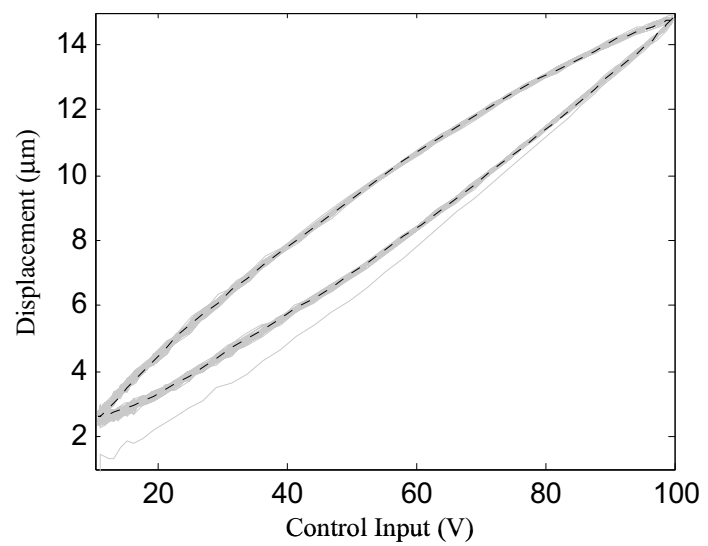

Figure 10. The lighter solid lines are the measured piezoelectric actuator response to a 10 $\mathrm{Hz}, 12.5 \mu \mathrm{m}$ p-p sinusoidal control input. The dark dotted line is the identified modified PI hysteresis model with 10 backlash operators $(n=9)$ and 4 dead-zone operators $(m=3)$. 
To find the hysteresis model parameters as shown in Fig. 10, we first have to measure experimentally the responses of the piezoelectric actuator to periodic control inputs. A good set of identification data is one that covers the entire operational actuation range of the piezoelectric actuator at the nominal operating frequency. Next decide the order of the PI operator $(n)$ and the saturation operator $(m)$, and set the threshold values $\vec{r}$ and $\vec{d}$ as described in the previous section. The weight parameters $\vec{w}_{h}$ and $\vec{w}_{s}$ are found by performing a least-squares fit of (9) to the measured actuator response, minimizing the error equation which is linearly dependent on the weights:

$$
E[x, z]\left(\vec{w}_{h}, \vec{w}_{s}, t\right)=\left[\vec{w}_{h}^{T} \vec{H}_{r}\left[x(t), \vec{y}_{0}\right](t)-\vec{w}_{s}^{T} \vec{S}_{d^{\prime}} z(t) .\right.
$$

Fig. 10 shows superposition of the identified modified PI hysteresis model on the measured piezoelectric actuator response, subjected to a sinusoidal control input.

\subsection{Inverse Modified Prandtl-Ishlinskii (PI) Operator}

The key idea of an inverse feedforward controller is to cascade the inverse hysteresis operator, $\Gamma^{-1}$, with the actual hysteresis which is represented by the hysteresis operator, $\Gamma$, to obtain an identity mapping between the desired actuator output $\hat{z}(t)$ and actuator response $z(t)$

$$
z(t)=\Gamma\left|\Gamma^{-1}[\hat{z}]\right|(t)=I[\hat{z}](t)=\hat{z}(t)
$$

The operation of the inverse feedforward controller is depicted in Fig.6.

The inverse of a PI operator is also of the PI type. The inverse PI operator is given by

$$
\Gamma^{-1}[\hat{z}](t)=\vec{w}_{h}^{T} \vec{H}_{r^{\prime}}\left[\vec{w}_{S}^{T} \vec{S}_{d^{\prime}}[\hat{z}], \vec{y}_{0}^{\prime}\right](t)
$$

where the inverse modified PI parameters can be found by

$$
\begin{gathered}
w_{h 0}^{\prime}=\frac{1}{w_{h 0}} ; w_{h i}^{\prime}=\frac{-w_{h i}}{\left(\sum_{j=0}^{i} w_{h j}\right)\left(\sum_{j=0}^{i-1} w_{h j}\right)}, i=1 \ldots n ; \\
r_{i}^{\prime}=\sum_{j=0}^{i} w_{h j}\left(r_{i}-r_{j}\right), \quad y^{\prime}=\sum_{j=0}^{i} w_{h j} y_{0 i}+\sum_{j=i+1}^{n} w_{h j} y_{0 j}, i=0 \ldots n ; \\
w_{s 0}^{\prime}=\frac{1}{w_{s 0}} ; w_{s i}^{\prime}=\frac{-w_{s i}}{\left(\sum_{j=0}^{i} w_{s j}\right)\left(\sum_{j=0}^{i-1} w_{s j}\right)}, i=1 \ldots m ; \\
d^{\prime}{ }_{i}=\sum_{j=0}^{i} w_{s j}\left(d_{i}-d_{j}\right), i=0 \ldots m ;
\end{gathered}
$$

\section{Rate-Dependent Phenomena}

Most, if not all, of the present mathematical models are defined rate-independent mathematically. This is too restrictive in real life. In this section, a rate-dependent hysteresis model is proposed. 


\subsection{Rate-dependent Hysteresis Slope}

In this section, an extension to the modified PI operator is proposed in order to also model the rate-dependent characteristics of the piezoelectric hysteresis is proposed. One of the advantages of the PI hysteresis model is that it is purely phenomenological; there are no direct relationships between the modeling parameters and the physics of the hysteresis. While the rate dependence of hysteresis is evident from Fig. 4, the sensitivity of actuator saturation to the actuation rate is not apparent. Hence, assuming that saturation is not ratedependent and hold the saturation weights, $\vec{w}_{s}$, as well as the threshold values, $\vec{r}$ and $\vec{d}$, constant a relationship between the hysteresis and the rate of actuation $\dot{x}(t)$ is constructed. The hysteresis slope (i.e., sum of the PI weights) at time $t$ as a rate-dependent function is

where

$$
\begin{gathered}
W_{h i}(\dot{x}(t))=\hat{W}_{h i}+f(\dot{x}(t)), i=1 \ldots n . ; \\
\dot{x}(t)=\frac{x(t)-x(t-T)}{T}, \dot{x}(0)=0 .
\end{gathered}
$$

\subsection{Rate-dependent Model Identification}

The piezoelectric actuator, subjected to periodic constant-rate or sawtooth control inputs. Measurements were made over a frequency band whose equivalent rate values cover the entire operational range of the actuation rates. For example, in an application tracking sinusoids of up to $12.5 \mu \mathrm{m} \mathrm{p}-\mathrm{p}$ in the band of 1 to $19 \mathrm{~Hz}$, the operational range of the actuation rate is from 0 to $746 \mu \mathrm{m} / \mathrm{s}$, which corresponds to the rate of $12.5 \mu \mathrm{m}$ p-p sawtooth waveforms of up to about $60 \mathrm{~Hz}$. PI parameter identification is then performed on each set of measured actuator responses. The sum of the hysteresis weights $W_{h i}, i=0 \ldots n$, of each identification is then plotted against the actuation rate $\dot{x}(t)$ and shown in Fig. 11 .

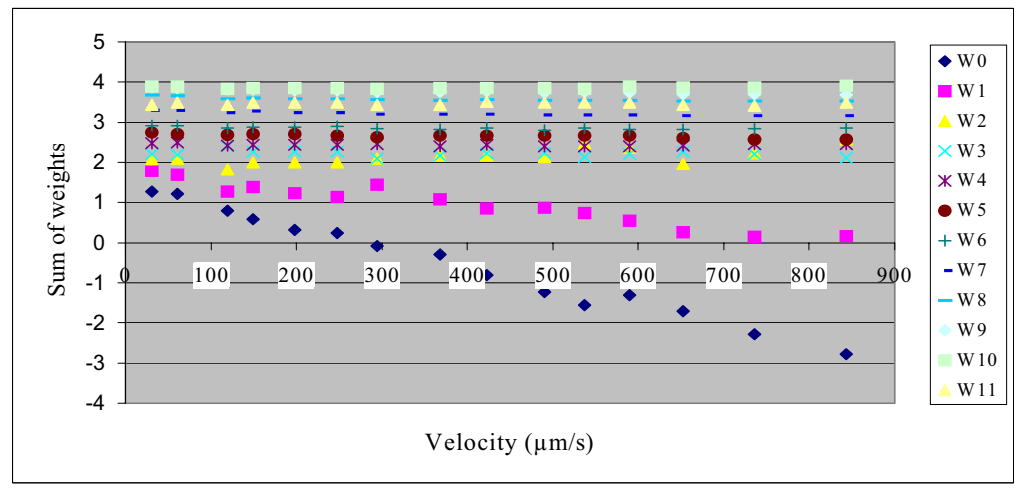

Figure 11. Plot of Sum of hysteresis weights against actuation rate

From Fig. 11, it can be seen that the hysteresis slope of the piezoelectric actuator can be modelled as linear to the velocity input with good approximation. Thus the rate-dependent hysteresis slope model would be:

$$
W_{h i}(\dot{x}(t))=\hat{W}_{h i}+c_{i} \dot{x}(t), i=0 \ldots n
$$


where $c_{i}$ is the slope of the best fit line through the $W_{h i}$ 's and the referenced slope, $\hat{W}_{h i}$, is the intercept of the best fit line with the vertical $W_{h}$ axis or the slope at zero actuation. The individual rate-dependent hysteresis weight values can be calculated from

$$
\begin{gathered}
w_{h i}(\dot{x}(t))=W_{h i}(\dot{x}(t))-W_{h(i-1)}(\dot{x}(t)), i=1 \ldots n ; \\
w_{h 0}(\dot{x}(t))=W_{h 0}(\dot{x}(t)) .
\end{gathered}
$$

4.3 Rate-dependent Modified Prandtl-Ishlinskii Operator

The rate-dependent modified PI operator is defined by

$$
z(t)=\Gamma[x, \dot{x}](t)=\vec{w}_{s}^{T} \vec{S}_{d}\left[\vec{w}_{h}^{T}(\dot{x}) \vec{H}_{r}\left[x, \vec{y}_{0}\right](t)\right.
$$

The inverse rate-dependent modified PI operator is also of the PI type:

$$
\Gamma^{-1}[\hat{z}](t)=\vec{w}_{h}^{T}(\dot{x}) \vec{H}_{r^{\prime}}\left\lfloor\vec{w}_{S}^{T} \vec{S}_{d^{\prime}}[\hat{z}], \vec{y}_{0}^{\prime} \mid(t) .\right.
$$

The inverse rate-dependent parameters can be found by (13), replacing $\vec{w}_{h}$ with the ratedependent $\vec{w}_{h}(\dot{x})$,

$$
\begin{gathered}
w_{h 0}^{\prime}(\dot{x}(t))=\frac{1}{w_{h 0}(\dot{x}(t))} ; \\
w_{h i}^{\prime}(\dot{x}(t))=\frac{-w_{h i}(\dot{x}(t))}{W_{h i}(\dot{x}(t)) W_{h(i-1)}(\dot{x}(t))}, i=1 \ldots n ; \\
r_{i}^{\prime}=\sum_{j=0}^{i} w_{h j}(\dot{x}(t))\left(r_{i}-r_{j}\right), \quad i=0 \ldots n ; \\
y^{\prime}{ }_{0 i}=\sum_{j=0}^{i} w_{h j}(\dot{x}(t)) y_{0 i}+\sum_{j=i+1}^{n} w_{h j}(\dot{x}(t)) y_{0 j}, i=0 \ldots n .
\end{gathered}
$$

\section{Motion Tracking Experiments}

Two motion tracking experiments were performed to demonstrate the rate-dependent feedforward controller. The first experiment compares the performance of the open loop feedforward controllers driven at fix frequencies. The rate-independent controller is based on the modified PI hysteresis model identified at the $10 \mathrm{~Hz}$ at $12.5 \mu \mathrm{m}$ peak to peak sinusoid. The second experiment is tracking a multi-frequency $(1,10$ and $19 \mathrm{~Hz})$ nonstationary motion profile.

\subsection{Experiment Setup}

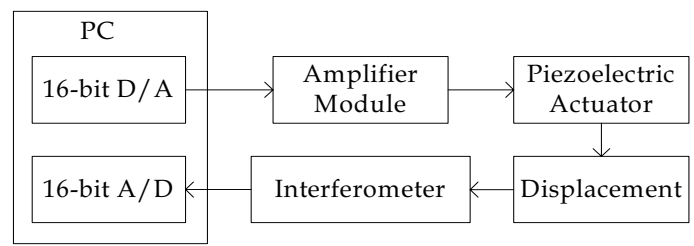

Figure 12. Experimental Architecture 
As seen from Fig. 12, a 16-bit D/A card is used to give out the necessary voltage, which is then passed through the amplifier (the gain is approximately 10). Given the voltage, the actuator will move and the interferometer will detect the displacement and convert it to analog voltage. Using a 16-bit A/D card, the PC reads in the displacement.

\subsection{Stationary Sinusoid Experiment}

The first experiment compares the performance of the rate-independent and rate-dependent modified PI models based open-loop feedforward controllers in tracking $12.5 \mu \mathrm{m} p-p$ stationary sinusoids at 1, 4, 7, 13, 16 and $19 \mathrm{~Hz}$. The tracking rmse and maximum error of each controller at each frequency are summarized in Table 1 and plotted in Fig. 13.

\begin{tabular}{|c|c|c|c|c|c|c|}
\hline \multirow[b]{2}{*}{ Freq. $(\mathrm{Hz})$} & \multicolumn{2}{|c|}{ Without Model } & \multicolumn{2}{|c|}{ Rate-independent } & \multicolumn{2}{|c|}{ Rate-dependent } \\
\hline & rmse $(\mu \mathrm{m})$ & $\max \varepsilon(\mu \mathrm{m})$ & rmse $(\mu \mathrm{m})$ & $\max \varepsilon(\mu \mathrm{m})$ & rmse $(\mu \mathrm{m})$ & $\begin{array}{c}\max \varepsilon \\
(\mu \mathrm{m})\end{array}$ \\
\hline 1 & 1.13 & 2.11 & 0.25 & 0.63 & 0.21 & 0.57 \\
\hline 4 & 1.12 & 2.07 & 0.19 & 0.67 & 0.16 & 0.46 \\
\hline 7 & 1.23 & 2.24 & 0.18 & 0.52 & 0.16 & 0.50 \\
\hline 10 & 1.19 & 2.26 & 0.14 & 0.46 & 0.17 & 0.47 \\
\hline 13 & 1.21 & 2.31 & 0.19 & 0.53 & 0.17 & 0.55 \\
\hline 16 & 1.30 & 2.49 & 0.27 & 0.59 & 0.17 & 0.53 \\
\hline 19 & 1.37 & 2.61 & 0.34 & 0.70 & 0.18 & 0.59 \\
\hline $\begin{array}{l}\text { Mean } \\
\pm \sigma\end{array}$ & $\begin{array}{c}1.22 \\
\pm 009\end{array}$ & $\begin{array}{c}2.30 \\
\pm 0.19\end{array}$ & $\begin{array}{c}0.23 \\
\pm 0.07\end{array}$ & $\begin{array}{r}0.59 \\
\pm 0.8\end{array}$ & $\begin{array}{c}0.18 \\
\pm 0.02\end{array}$ & $\begin{array}{c}0.52 \\
\pm 0.05\end{array}$ \\
\hline
\end{tabular}

The rmse's and max errors are the mean results over a set of three 5-second (5000 data points) experiments.

Table 1. Measured Performance of the Rate-Independent and Rate-Dependent Inverse Feedforward Controllers
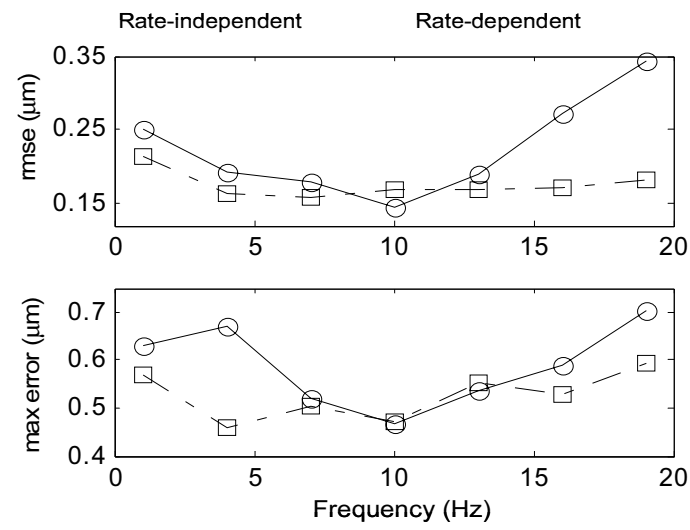

Figure 13: Experimental tracking results of different controllers for stationary $12.5 \mu \mathrm{m}$ at 10 $\mathrm{Hz}$ 
As shown in Fig. 13, at $19 \mathrm{~Hz}$, the tracking rmse of the rate-independent controller is almost double that of the rate-dependent controller and will continue to worsen as the frequency increases. Fig. 14 shows the results of the different controllers. Fig. 14(a) plots the hysteretic response of the piezoelectric actuator with a proportional controller. Fig. 14(b) and Fig. 14(c) presents the tracking ability of the rate-independent and rate-dependent inverse feedforward controllers respectively. The rate-independent controller is based on the modified PI hysteresis model identified at the same $10 \mathrm{~Hz}, 12.5 \mu \mathrm{m}$ p-p sinusoid.

Both the rate-independent and rate-dependent controllers significantly reduced the tracking error due to the piezoelectric hysteretic behaviour. However, the tracking accuracy of the rate-independent controller deteriorates when the frequency deviates from $10 \mathrm{~Hz}$. Meanwhile, the rate-dependent controller maintained a smaller rmse and maximum error.

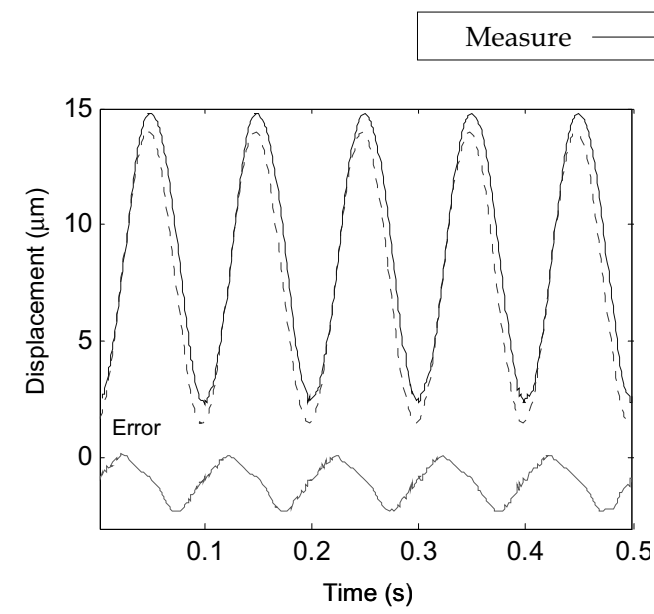

(a) Without Controller

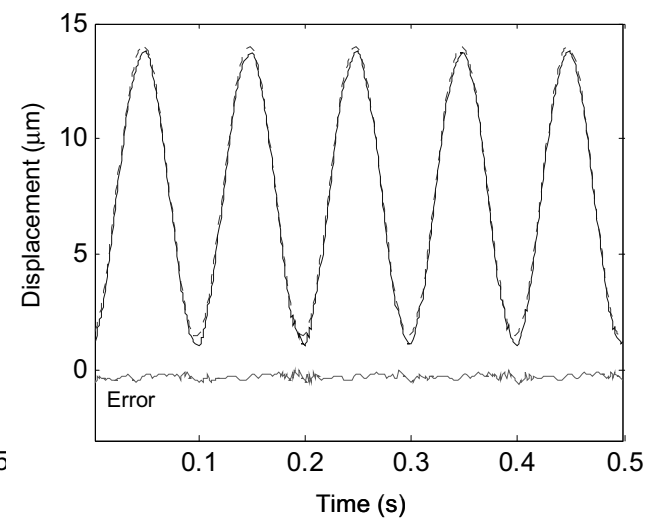

(b) Rate-Independent Controller

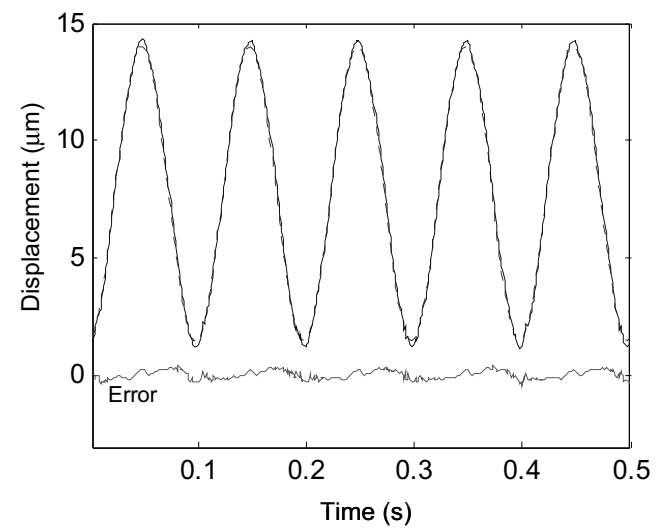

(c) Rate-Dependent Controller

Figure 14. Rmse and maximum errors of the rate-independent and rate-dependent controllers in tracking $12.5 \mu \mathrm{m}$ p-p stationary sinusoids at different frequencies 


\subsection{Multi-Frequency Nonstationary Experiment}

The second experiment is an experiment to test the ability of the controllers to track a multifrequency nonstationary motion profile. Both the feedforward controllers do improve the tracking capability. However, the rate-dependent controller did noticeably better. The result is shown in Fig. 15 and summarised in table 2.

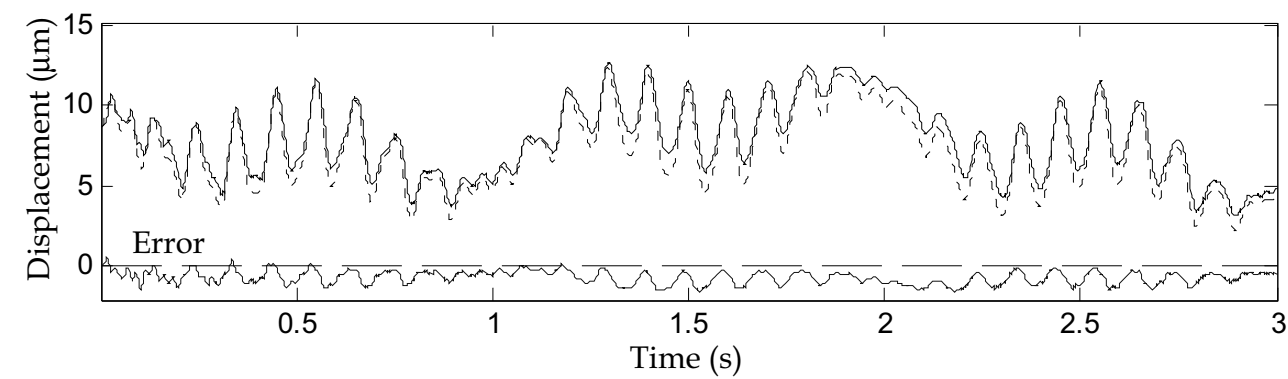

(a)

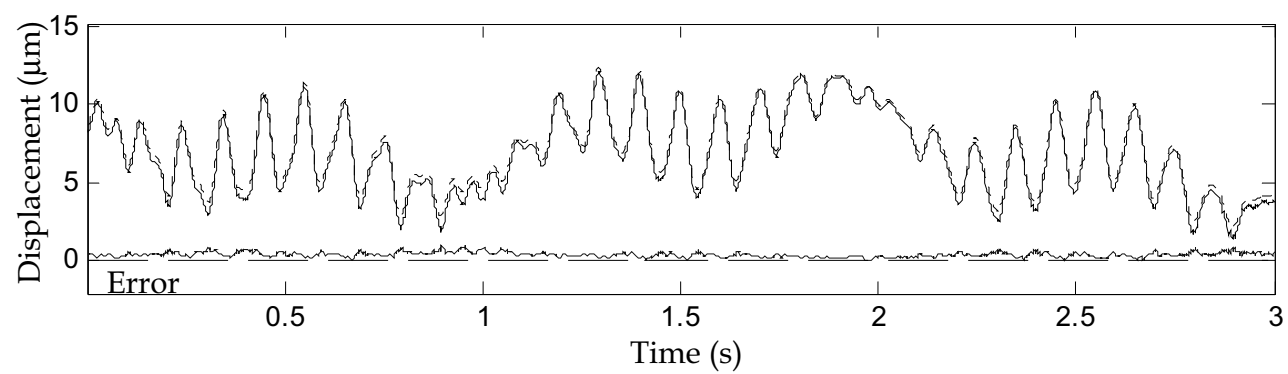

(b)

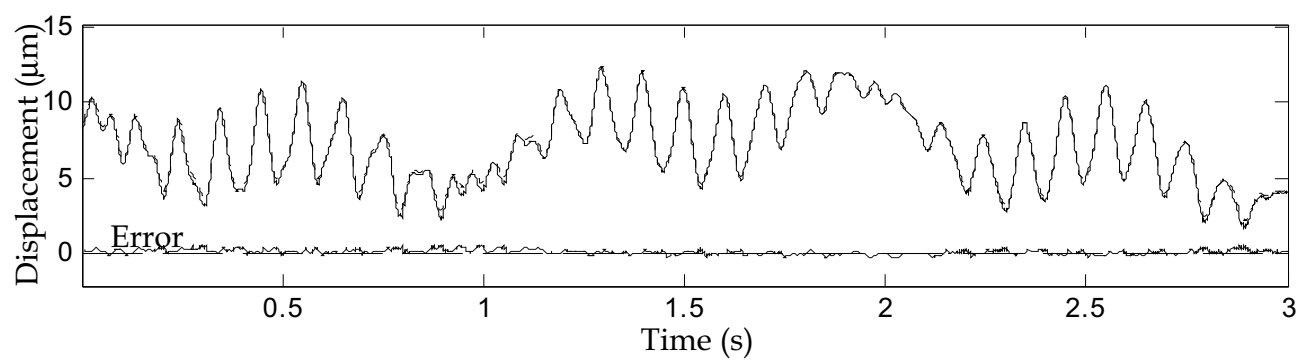

(c)

Figure 15. Experimental open-loop tracking results of a multi-frequency, nonstationary dynamic motion profile. The motion profile is made up of superimposed modulated 1, 10, and $19 \mathrm{~Hz}$ sinusoids with time-varying amplitudes. The rate-independent controller is based on the modified PI hysteresis model identified at the same $10 \mathrm{~Hz}, 12.5 \mu \mathrm{m}$ p-p sinusoid. Transient error is observed for the rate-independent controller in the first 2 seconds.(a) Without compensation. (b) Rate-independent controller. (c) Rate-dependent controller 


\begin{tabular}{lccc}
\hline & Without model & Rate-independent & Rate-dependent \\
\hline $\begin{array}{l}\text { rmse } \pm \sigma \\
(\mu \mathrm{m})\end{array}$ & $1.02 \pm 0.07$ & $0.31 \pm 0.03$ & $0.15 \pm 0.003$ \\
$\frac{\text { rmse }}{\mathrm{p} \text { - } \text { amplitude }}(\%)$ & 9.2 & 2.8 & 1.4 \\
$\begin{array}{l}\text { max error } \pm \sigma \\
(\mu \mathrm{m})\end{array}$ & $1.91 \pm 0.08$ & $0.89 \pm 0.04$ & $0.59 \pm 0.06$ \\
$\frac{\max \text { error }}{\mathrm{p} \text { - } \text { pamplitude }}(\%)$ & 17.3 & 8.0 & 5.3
\end{tabular}

The rmse and max errors are the mean results over a set of seven 5-second (5000 data points) experiments.

Table 2. Measured Performance of the Rate-Independent and Rate-Dependent Inverse Feedforward Controllers in Tracking Multi-Frequency (1, 10 and $19 \mathrm{~Hz})$ Nonstationary Signals

The rate-dependent controller registers a tacking rmse less than half of that of the rateindependent controller. Maximum tracking errors for both controllers occur in the transient phase. This might explain why the improvement in maximum error with the rate-dependent controller is not as large as the improvement in rmse. One limitation of all PI-type hysteresis models is that singularity occurs when the first PI weight is 0 as seen in equation (13). Also, when the slope is negative, the inverse hysteresis loading curve violates the fundamental assumption that it should be monotonically increasing. Thus, the inverse model will be lost. In order to maintain a good tracking accuracy for high velocity by having small threshold intervals, a method to solve the singularity problem is proposed in the next section.

\section{Using a different Domain to solve Singularity Problem}

The PI operator, while being able to model the hysteresis behaviour of a piezoelectric actuator well, has one major inadequacy: the inverse of the operator does not exist when the slope of the hysteretic curve is not positive definite, i.e. singularity occurs when the PI weights $\leq 0$. Such ill conditioned situations arise when the piezoelectric actuators are used to actuate heavy loads or when operating at high frequency. Another possible situation for ill condition is when small intervals between the threshold values are used. Presently, most people avoid this problem by having larger intervals between the threshold values. However, this is not solving the problem and resulted in higher error around the turning point.

This section presents how the authors managed to overcome this problem by mapping the hysteresis through a linear transformation onto another domain, where the inversion would be better behaved. The inverse weights are evaluated in this domain and are subsequently used to compute the inverse hysteresis model, which is to be used in the feedforward controller, before the inverse model is transformed back to the original domain. The singularity problem is first illustrated, followed by the solution to map the ill-conditioned hysteresis onto a singularity-free domain.

\subsection{Illustration of Problem}

As seen in Fig. 5, using the inverse as a feedforward controller linearizes the response. Unfortunately, to use the hysteresis model, the operating frequency must not be too high as the hysteresis non-linearity will become more severe and like most models, the classical 
Prandtl-Ishlinskii model is also unable to function as a feedforward controller when the largest displacement does not occur at the highest input signal (Fig. 6). The inverse model equation (13) fails when the convex curve is encountered.

Most systems can be approximated as a spring mass damper system. When driven at high velocity, the actuator/mechanism has a high momentum at the turning point, especially if a rapid change is made. The large momentum tends to keep the system in motion and the large momentum results in the convex curve. Similar explanation is applicable for large loads.

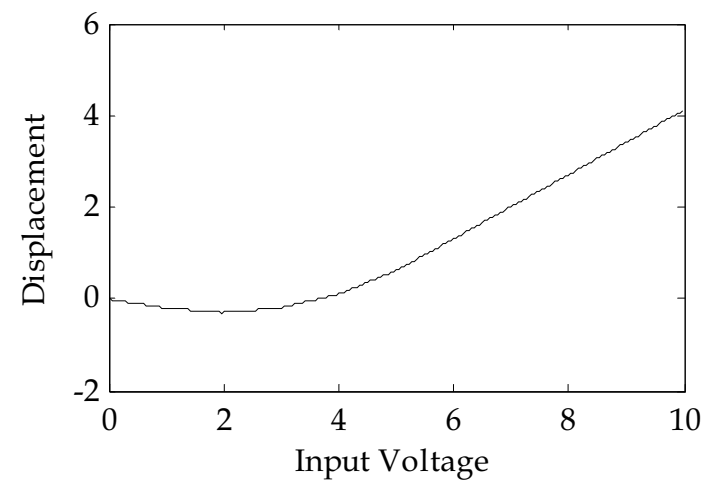

Figure 16. Loading Curve of Hysteresis example involving negative gradient

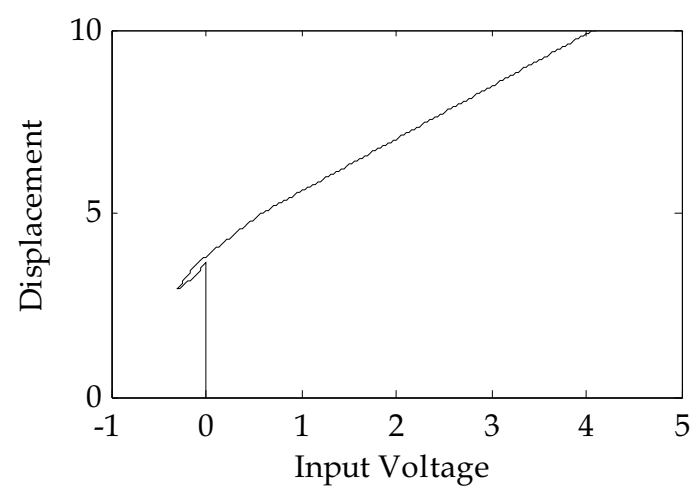

Figure 17. Inverse Loading Curve of example to illustrate failure of PI inverse operator when negative gradient is encountered

There is also an inevitable trade-off between modeling accuracy and inversion stability. The modeling of the hysteretic loop gets better with the number of backlash operators used in the modeling. However, as the piecewise continuous interval represented by each backlash operator shrinks, there is a greater chance for the reciprocal of the PI weights to be ill conditioned, especially at the hysteretic curve turning points. An example to show that the inverse model equation (13) fails when the convex curve is encountered is illustrated here. Given weights of $\vec{w}_{h}^{T}=\left[\begin{array}{llllll}0.2 & 0.1 & 0.2 & 0.2 & 0.2 & 0.2\end{array}\right]$ and $\vec{r}=\left[\begin{array}{lllll}0 & 1 & 2 & 3 & 4\end{array}\right]$ for an application where the amplitude of the periodic input voltage is $10 \mathrm{~V}$. The loading curve is shown in Fig. 16. 
Applying equation (13) to the get the inverse PI parameters, we obtain $\vec{w}_{h}^{T}=\left[\begin{array}{ll}-5 & -5 \\ 20 & -\end{array}\right.$ $6.6667-1.333-0.5714]$ and $\vec{r}^{\prime}=\left[\begin{array}{lllll}0 & -0.2 & -0.3 & 0.1 & 0.6\end{array}\right]$. Fig. 17 illustrates the inverse curve that will be obtained using equation (13). The two graphs are not a reflection of each other along the 45 degrees line. This simple proof clearly illustrates that equation (13) has failed as an inverse function when the condition of positive gradient is not met. Zero gradient is not demonstrated in this example as it is clear that the reciprocal of 0 is a singular point.

\subsection{Obtaining Inverse Model in a Different Domain}

\subsubsection{Intuition of Proposed Method}

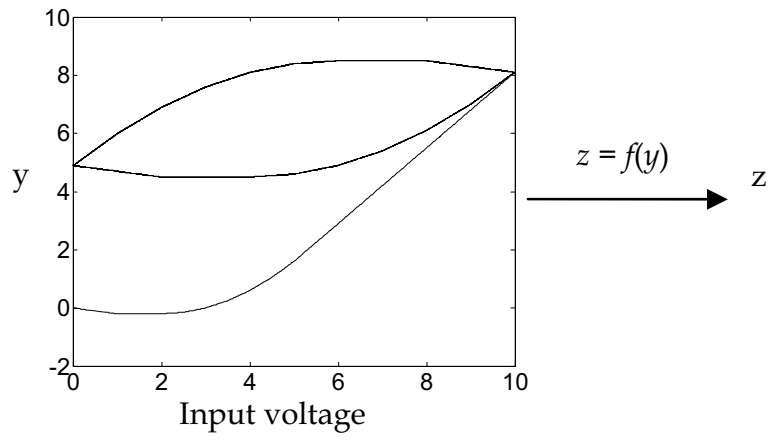

Figure 18. Transformation
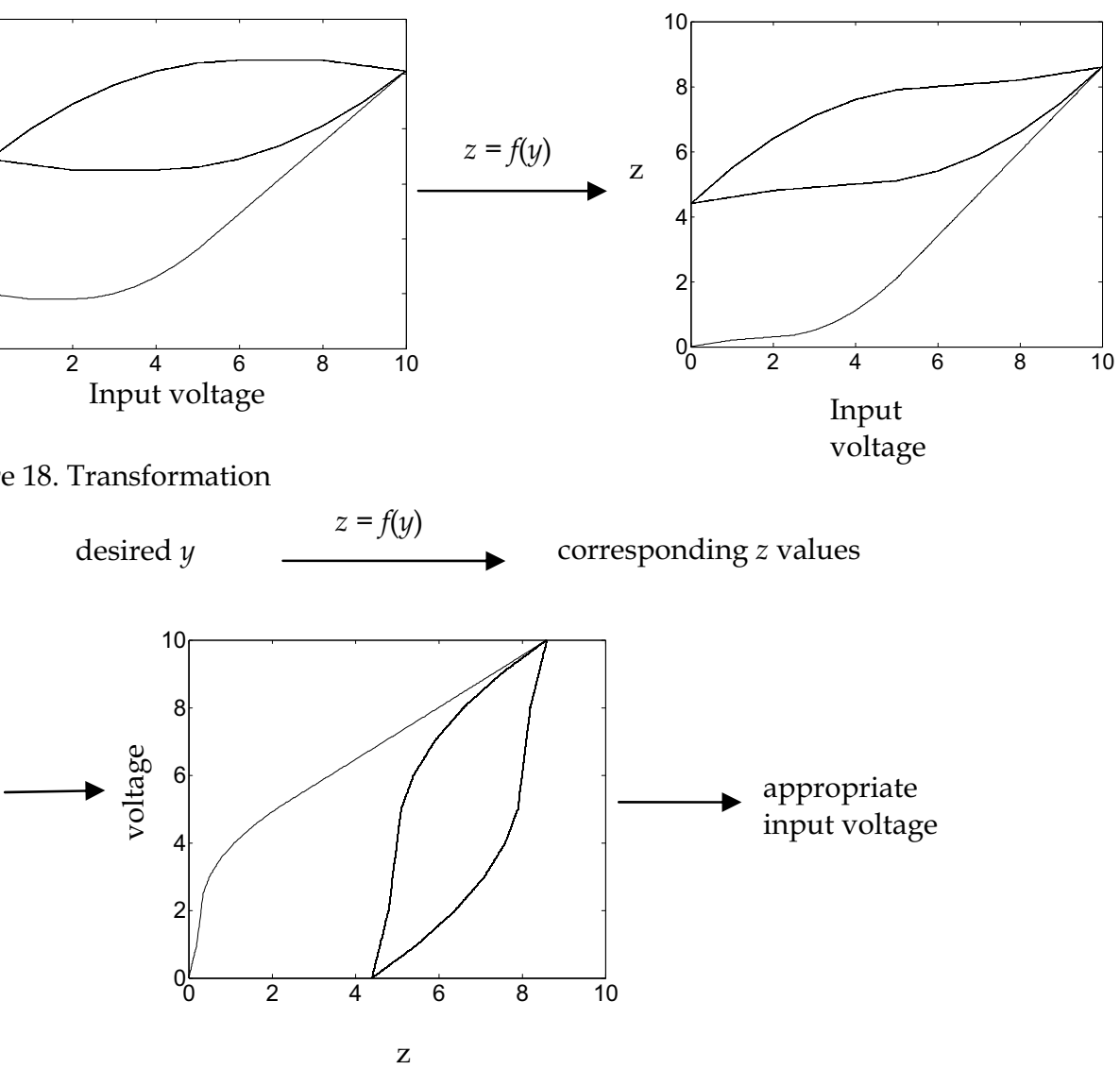

Figure 19. Method to Obtain Appropriate Input Voltage

With the singularity problem, the author came up with the idea to model the hysteresis in an alternative domain when the inverse of the PI model fails in a situation like the largest displacement not occurring at the highest input signal (Fig. 6). A transformation is used to map $y$ (the original hysteretic displacement values) to $z$, which has no singular points as shown in Fig. 18. The inverse model can now be obtained in the new domain. The 
appropriate input voltages can now be obtained using the inverse model found in the new domain as shown in Fig. 19.

The saturation operator is just another transformation and thus can be ignored for the time being. The inverse of the saturation operator can be applied after the inverse of the singularity-free model.

The desired value displacement $y$ is first passed through the transformation function to obtain the corresponding new domain $\mathrm{z}$ value. This $\mathrm{z}$ value is then passed through the inverse model obtained in the new domain to get the appropriate input voltages.

\subsubsection{Obtaining the Inverse Model in a different Domain}

Although the inverse of the PI model fails, PI model can still describe the pneumonia path like figure 4 . The PI parameters for the ill-conditioned hysteresis can be obtained as shown in section 2. Recall that equation (2) can describe the hysteresis.

$$
y(t)=\vec{w}_{h}^{T} \vec{H}_{r}\left[x, \vec{y}_{0}\right](t)
$$

Using least square method, $\vec{w}_{h}^{T}$ is be obtained. To illustrate the transformation, nine points $\left(x_{0}\right.$ to $\left.x_{8}\right)$ are labelled in Fig. 20. Negative gradient for the loading curve occurs between $x_{0}$ and $x_{1}$ while 0 gradient is between $x_{1}$ and $x_{2}$. In the hysteresis loop, region between $x_{3}$ to $x_{4}$ and $x_{6}$ to $x_{7}$ has negative gradient while $x_{4}$ to $x_{5}$ and $x_{7}$ to $x_{8}$ contain gradient value 0 . The labelled points $x_{0}$ to $x_{8}$ can be calculated using:

$$
\begin{aligned}
& x_{0}=0 ; \\
& \text { If } \sum_{j=1}^{i} w_{h_{j}}=0 \text { exists, } x_{1}=r_{i} ; x_{2}=r_{i+1} \\
& \text { otherwise, } x_{1}=x_{2}=r_{\max } \text { where } \sum_{j=1}^{\max } w_{h_{j}}>0 \\
& x_{3}=2 r_{\max } ; \\
& x_{4}=x_{3}-2 x_{1} ; \\
& x_{5}=x_{3}-2 x_{2} ; \\
& x_{6}=0 ; \\
& x_{7}=x_{6}+2 x_{1} ; \\
& x_{8}=x_{6}+2 x_{2} ;
\end{aligned}
$$

To obtain the points $\mathrm{a}_{0}$ to $\mathrm{a}_{8}, \mathrm{x}_{0}$ to $\mathrm{x}_{8}$ are substituted into equation (4).

The transformation function $f(x)$ as shown in Fig. 19 is a function that changes the weights of the PI hysteresis model. The weights of the transformed hysteresis are obtained via:

$$
w_{2 h_{i}}= \begin{cases}-\sum_{j=1}^{i} w_{h_{j}}-\sum_{j=1}^{i-1} w_{2 h_{j}} & , \sum_{j=1}^{i} w_{h_{j}}<0 \\ c-\sum_{j=1}^{i-1} w_{2 h_{j}} & , \sum_{j=1}^{i} w_{h_{j}}=0 \\ \sum_{j=1}^{i} w_{h_{j}}-\sum_{j=1}^{i-1} w_{2 h_{j}} & , \sum_{j=1}^{i} w_{h_{j}}>0\end{cases}
$$


where $\mathrm{c}$ is a positive non-zero constant to force the transformed gradient to be positive nonzero number. Fig. 21 shows the relationship of $\mathrm{z}$ and $\mathrm{y}$ after passing through the transformation function. The constants $a_{i}$ and $b_{i}$ are the corresponding $y$ and $z$ values respectively to input voltage $x_{i}$.

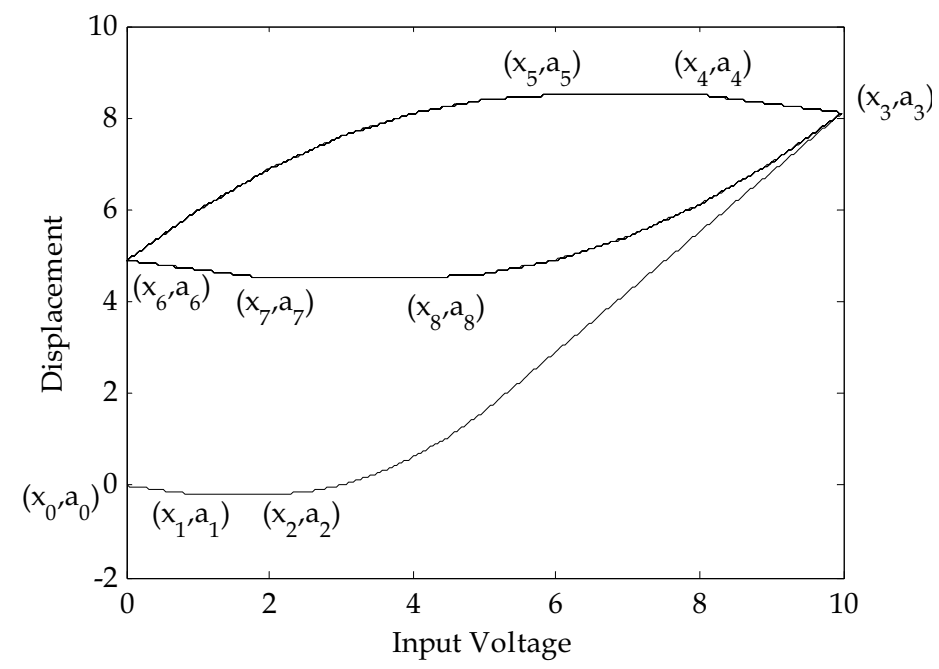

Figure 20: Graph of an ill-conditioned Hysteresis with points $\mathrm{x}_{0}$ to $\mathrm{x}_{8}$ labelled

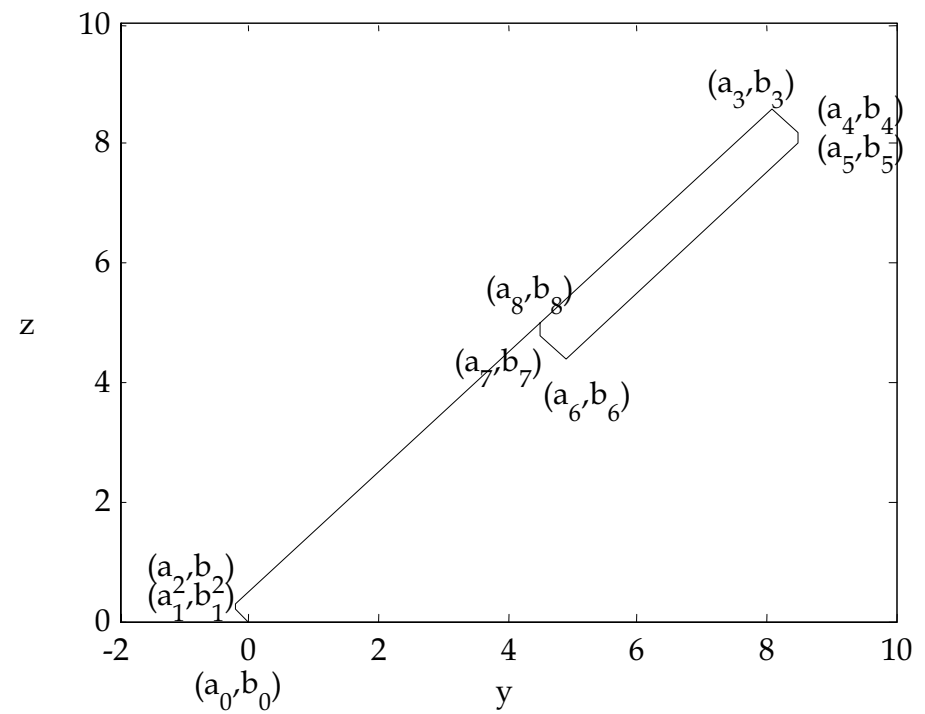

Figure 21: Relationship of $\mathrm{z}$ (new domain) with y (real displacement) 
Because of the way the transformation function is formed, all the gradients of the line are 1, 1 or infinite. Points $b_{0}$ to $b_{8}$ are obtain using (24).

$$
\begin{aligned}
& b_{0}=0 ; \\
& b_{1}=-a_{1} ; \\
& b_{2}=a_{1}+c \times\left[x_{2}-x_{1}\right] ; \\
& b_{3}=b_{2}+\left[a_{3}-a_{2}\right] ; \\
& b_{4}=b_{3}-\left[a_{4}-a_{3}\right] ; \\
& b_{5}=b_{4}-2 c \times\left[x_{2}-x_{1}\right] ; \\
& b_{6}=b_{5}+\left[a_{6}-a_{5}\right] ; \\
& b_{7}=b_{6}-\left[a_{7}-a_{6}\right] ; \\
& b_{8}=b_{7}+2 c \times\left[x_{2}-x_{1}\right] ;
\end{aligned}
$$

With these points, the relationship between $z$ to $y$ is in table 3 .

\section{Simulation and Experimental Results of Transformation Method}

\subsection{Simulation}

This section demonstrates how the transformation function is used to help the reader in applying the equations shown to their applications.

The actual ill conditioned hysteresis of the system is first obtained and modelled using Prandtl-Ishlinskii operator. The hysteresis curve is then mapped onto another domain using the transformation function illustrated in section 6. A well-conditioned hysteresis is obtained as seen in Fig.18. The inverse parameters of the well-conditioned hysteresis curve in the new domain are obtained using (13) and the inverse model is obtained.

After obtaining the inverse function in the new domain, the desired y values are passed through the transformation to obtain the desired $\mathrm{z}$ values using table 4 , starting with $\mathrm{A}=0$, $B=0$ and $C=0$. The desired $z$ values are then passed through the inverse Prandtl-Ishlinskii model to obtain the required input $x$. An example is illustrated in Fig. 22, where the red graph is the hysteresis and blue graph is the inverse curve.

\begin{tabular}{|l|l|l|}
\hline$y$ value & Corresponding $z$ value & Equation \\
\hline $\mathrm{a}_{0}$ to $\mathrm{a}_{1}$ & $z=-y$ & $(25)$ \\
\hline $\mathrm{a}_{1}$ to $\mathrm{a}_{2}$, & $z=b_{2}$, any value between $b_{1}$ to $b_{2}$ & $(26)$ \\
\hline $\mathrm{a}_{2}$ to $\mathrm{a}_{3}$, & $z=y+b_{2}-a_{2}$ & $(27)$ \\
\hline $\mathrm{a}_{3}$ to $\mathrm{a}_{4}$ & $z=-y+b_{3}+a_{3}$ & $(28)$ \\
\hline $\mathrm{a}_{4}$ to $\mathrm{a}_{5}$ & $z=b_{5}$, any value between $b_{4}$ to $b_{5}$ & $(29)$ \\
\hline $\mathrm{a}_{5}$ to $\mathrm{a}_{6}$ & $z=y+b_{5}-a_{5}$ & $(30)$ \\
\hline $\mathrm{a}_{6}$ to $\mathrm{a}_{7}$ & $z=-y+b_{6}+a_{6}$ & $(31)$ \\
\hline $\mathrm{a}_{7}$ to $\mathrm{a}_{8}$ & $z=b_{8}$, any value between $b_{7}$ to $b_{8}$ & $(32)$ \\
\hline
\end{tabular}

Table 3. Relation between the two domains 


\begin{tabular}{|c|c|c|c|}
\hline Condition (1) & Condition (2) & Equation & Setting \\
\hline \multirow{2}{*}{$A^{\prime} B^{\prime} C^{\prime}$} & $|y(t)|<\left|a_{1}\right| \& \& y(t)<y(t-1)$ & $(25)$ & \\
\hline & otherwise & (26) & \\
\hline \multirow{2}{*}{$A^{\prime} B^{\prime} C$} & $y(\mathrm{t})=a_{2}$ & $(26)$ & \\
\hline & otherwise & $(27)$ & $\mathrm{B}=1$ \\
\hline \multirow{2}{*}{$A^{\prime} B C$} & $y(t) \leq a_{3}$ & $(27)$ & \\
\hline & otherwise & $(28)$ & $\mathrm{A}=1$ \\
\hline \multirow{2}{*}{$A B C$} & $y(\mathrm{t}) \leq a_{4}$ & $(28)$ & \\
\hline & otherwise & $(29)$ & $\mathrm{B}=0$ \\
\hline \multirow{2}{*}{$A B^{\prime} C$} & $y(t)=a_{5}$ & $(29)$ & \\
\hline & otherwise & $(30)$ & \\
\hline \multirow{2}{*}{$A B^{\prime} C^{\prime}$} & $y(t) \geq a_{6}$ & (30) & \\
\hline & otherwise & (31) & $B=1$ \\
\hline \multirow{2}{*}{$A B C^{\prime}$} & $y(\mathrm{t}) \geq a_{7}$ & $(31)$ & \\
\hline & otherwise & $(32)$ & $A=0$ \\
\hline \multirow{2}{*}{$A^{\prime} B C^{\prime}$} & $y(t)=a_{8}$ & $(32)$ & \\
\hline & otherwise & $(27)$ & \\
\hline
\end{tabular}

Table 4. Equations to obtain $y$ values

The value of $\mathrm{C}$ is as follows:

$$
C= \begin{cases}0 & , y(t)<y(t-T) \\ 1 & , y(t)>y(t-T)\end{cases}
$$

As shown in Fig. 22, the final inverse graph (blue) is a reflection of the hysteresis graph (red) along the line $y=x$. This clearly illustrates the ability of the transformation function to obtain the inverse of the hysteresis curve. The transformation function has no effect on wellconditioned hysteresis graphs as $\mathrm{x}_{2}=\mathrm{x}_{1}=\mathrm{x}_{0}$.
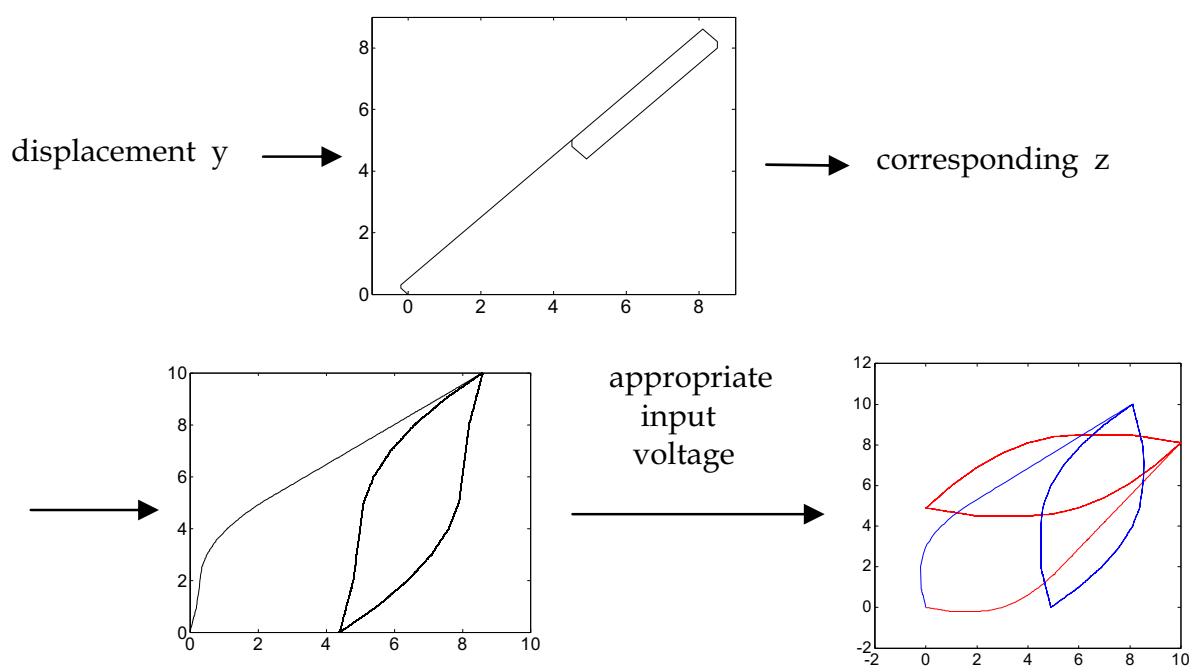

Figure 22. Simulation to illustrate that the inverse can be obtained with desired y as input 


\subsection{Experimental Results}

The experiment setup is as described in section 5.1. Three types of experiments were carried out. The first set of experiments is $8 \mathrm{~Hz}$ triangular wave. Triangular wave is used because of its constant velocity. This is followed by varying amplitude linear motion with varying velocity to demonstrate the capability to model rate-dependent. The last experiment is a varying frequency with varying amplitude sinusoidal wave.

The same model is being used for both with and without mapping. The first experiment's desired displacement is a triangular wave with the velocity high enough for the first weight to go into the negative region.

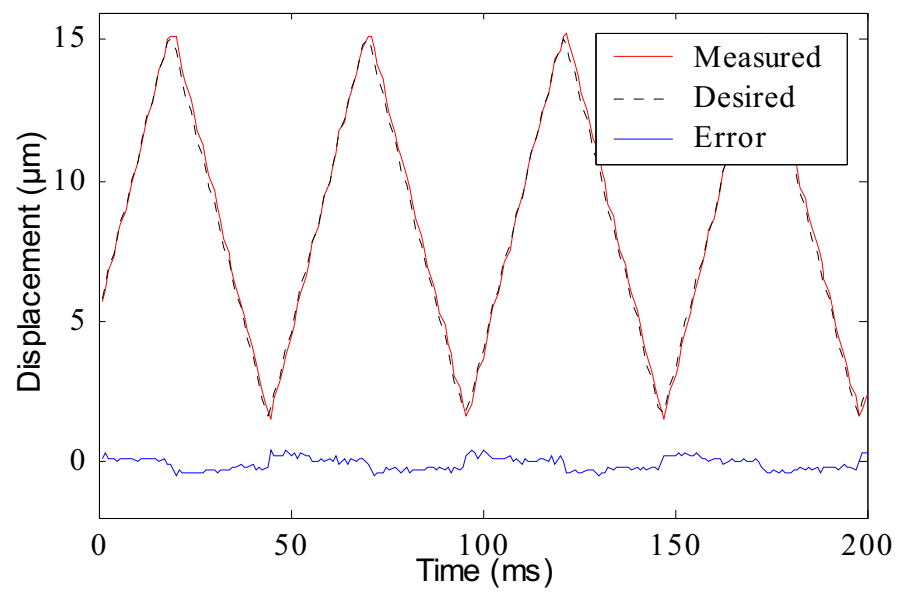

Figure 23. Triangular Wave Without Mapping

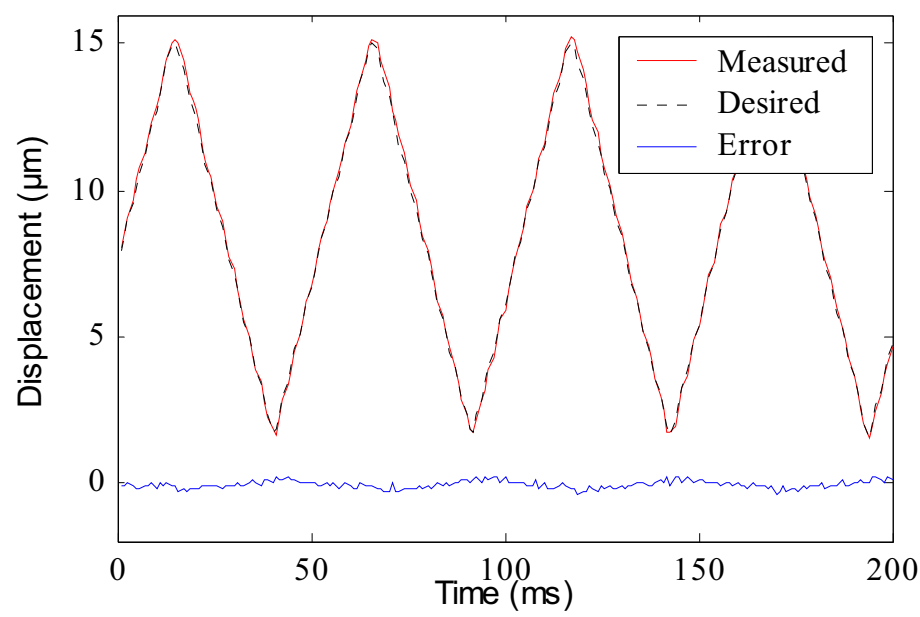

Figure 24. Triangular Wave With Mapping 


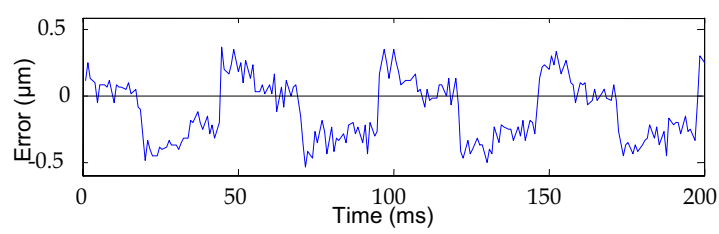

Figure 25. Exploded View of Error of Triangular Wave Without Mapping

\begin{tabular}{|c|c|c|c|c|}
\hline & \multicolumn{2}{|c|}{ Triangular } & Non-Periodic linear motion \\
\hline & $\begin{array}{c}\text { Without } \\
\text { Mapping }\end{array}$ & $\begin{array}{c}\text { With } \\
\text { Mapping }\end{array}$ & $\begin{array}{c}\text { Without } \\
\text { Mapping }\end{array}$ & $\begin{array}{c}\text { With } \\
\text { Mapping }\end{array}$ \\
\hline rmse $(\mu \mathrm{m})$ & 0.2580 & 0.1544 & 0.2267 & 0.1328 \\
\hline rms error reduction & \multicolumn{2}{|c|}{$40.1 \%$} & \multicolumn{2}{c|}{$41.4 \%$} \\
\hline Max. Error $(\mu \mathrm{m})$ & 0.5478 & 0.4222 & 0.4959 & 0.3473 \\
\hline
\end{tabular}

Table 5. Experimental Results on Control of Piezoelectric Actuator

From Fig. 25, it can be clearly seen that the error has a general shape of a square wave. It has a general offset of overshoot when the desired displacement is increasing and an offset of undershoot when the displacement is decreasing. With mapping, this overshoot or undershoot are removed. Thus it can be clearly seen that the error in Fig. 23 (without mapping) is higher than the error in Fig. 24 (with mapping) and the rms error is greatly reduced by $40.1 \%$. This proved that it is the singularity problem in the inverse expression that is creating the problem and not the hysteresis model.

Similar findings were obtained with non-periodic linear motion. Fig. 26 and Fig. 27 show the result of without and with mapping respectively. As seen from Fig. 26, like Fig. 23, there is also a constant overshoot or undershoot in the error depending on the direction of actuation. With mapping, the offsets are removed and the rms error is reduced by $41.4 \%$. Table 5 is a summary of the results.

Fig. 28 is an experiment to show that the model is also valid for non-periodic varying sinusoidal waves and the rms error obtained is $0.14436 \mu \mathrm{m}$. These figures demonstrate that the model is able to model non-periodic motion.

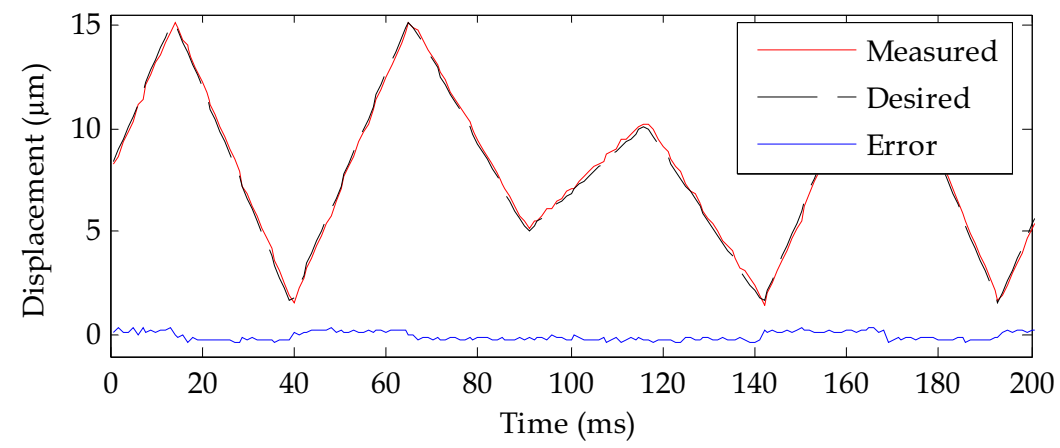

Figure 26. Non-Periodic Linear Motion Without Mapping 


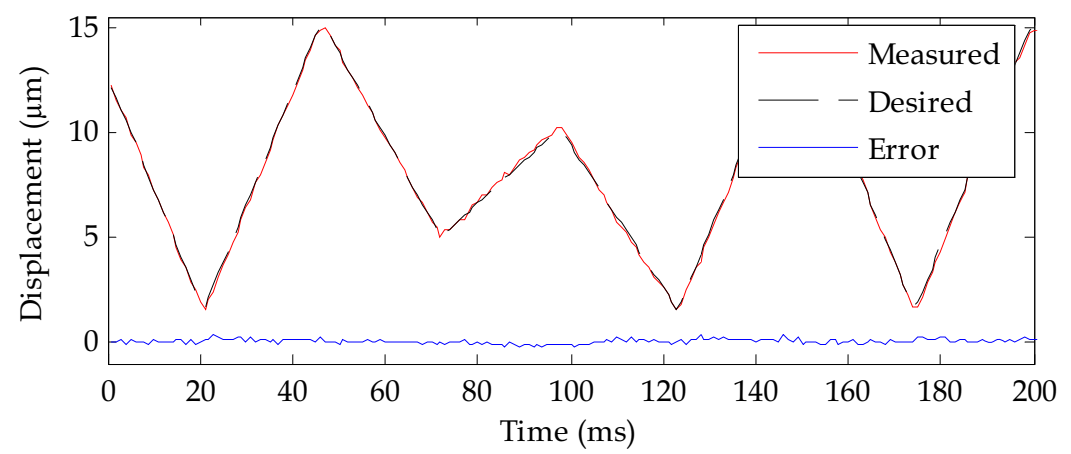

Figure 27. Non-Periodic Linear Motion With Mapping

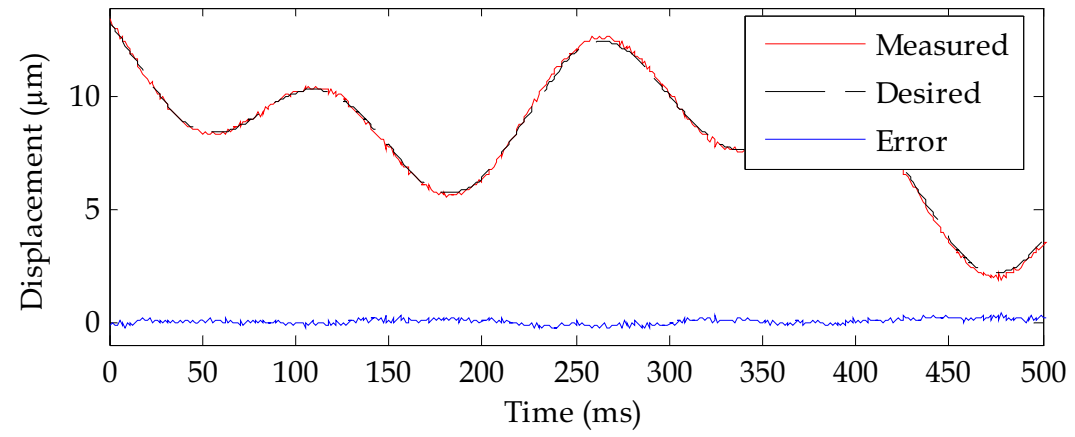

Figure 28. Superposition of a few different frequencies of sinusoidal waves

\section{Conclusion}

Human hand is the best manipulator available. However, its manual positioning accuracy is limited due to the involuntary tremor motion. Most of the current methods are non-active compensating methods like filtering of the tremor motion. Examples include the master and slave systems and the third hand. In this chapter, since humans are in possession of a high dexterity manipulator with an unbeatable user interface, instead of replacing the human hand with a robotic manipulator, active compensation of the physiological tremor is proposed.

Piezoelectric actuators are used to compensate the tremor motion. Two main contributions are made in this chapter, namely: (1) a rate-dependent feedforward controller; and (2) a solution to the inverse of an ill-conditioned hysteresis.

Physiological tremor is modulating frequency behaviour. Although the tremor is near sinusoidal at 8-12 Hz, tremor is non-periodic. Active compensation of active physiological tremor requires a zero phase rate-dependent controller. In this chapter, a rate-dependent Prandtl-Ishlinskii hysteresis model has been proposed. With this rate-dependent feedforward controller, piezoelectric actuators can now be used to compensate non-periodic 
disturbance. With velocity as one of its input, the rate-dependent feedforward controller is now able to account for the non-periodic signals.

The feedforward controller is obtained through phenomenal modelling. Although the model obtained is specific to the hardware and setup, the method can be applied to other applications because underlying physics knowledge is not required. The feedforward controller is implemented in an open loop system. Some advantages of open loop control includes no stability problem faced by controllers and lower cost as sensors are not required for the feedback information.

Traditionally, people tried to control the conditions such that the ill-conditioned hysteresis situation is avoided. This is not solving the problem and will result in higher error. A method to overcome this problem has also been demonstrated. This is achieved by mapping the ill-conditioned hysteresis onto a different domain to obtain a well conditioned hysteresis. The inverse is then obtained in this new domain. The equations relating the two domains are also given in this chapter.

\section{References}

Abidi, K.; Sabanovic, A. \& Yesilyurt, S. (2004). Sliding Mode Control Based Disturbance Compensation and External Force Estimation for a Piezoelectric Actuator, IEEE Int. Workshop on Advance Motion Control, pp. 529-534, Japan, March 2004.

Ang, W. T.; Garmon, F. A.; Khosla, P. K. \& Riviere, C. N. (2003). Modeling Rate-dependent Hysteresis in Piezoelectric Actuators, IEEE/RSJ Int. Conf. Intelligent Robots and Systems (IROS), pp. 1975-1980, Las Vegas, Nevada, Oct., 2003.

Chen, B. M.; Lee, T. H.; Hang, C. C.; Guo, Y. \& Weerasooriya, S. (1999). An $\mathrm{H}_{\infty}$ Almost Disturbance Decoupling Robust Controller Design for a Piezoelectric Bimorph Actuator with Hysteresis. IEEE Transactions on Control Systems Technology, vol. 7 No. 2, (March 1999), pp. 160-174.

Choi, D. Y. \& Riviere, C. N. (2005). Flexure-Based Manipulator for Active Handheld Microsurgical Instrument, 27th Annual International Conference of the IEEE Engineering in Medicine and Biology Society (EMBS), pp. 5085-5088, Shanghai, China, Sept. 2005.

Choi, G. S.; Kim, H. S. \& Choi, G. H. (1997). A Study on Position Control of Piezoelectric Actuators, IEEE Int. Symposium on Industrial Electronics, pp. 851-855, Guimaraes, Portugal, 1997.

Cruz-Hernandez, J. M. \& Hayward, V. (1998). Reduction of Major and Minor Hysteresis Loops in a Piezoelectric Actuator, IEEE Conference on Design \& Control, pp. 43204325, Tampa, Florida USA, 1998.

Cruz-Hernandez, J. M. \& Hayward, V. (2001). Phase Control Approach to Hystersis Reduction. IEEE Transactions on Control Systems Technology, vol. 9, No. 1, (Jan. 2001), pp. 17-26.

Furutani, K.; Urushibata, M. \& Mohri, N. (1998) Improvement of Control Method for Piezoelectric Actuator by Combining Induced Charge Feedback with Inverse Transfer Function Compensation, IEEE Int. Conf. On Robotics \& Automation, pp. 1504-1509, Leuven, Belgium, May 1998.

Goldfarb, M. \& Celanovic, N. (1996). Behavioral Implications of Piezoelectric Stack Actuators for Control of Micromanipulation, IEEE Int. Conf. on Robotics $\mathcal{E}$ Automation, pp. 226-231, Minneapolis, Minnesota, USA, 1996. 
Goldfarb, M. \& Celanovic, N. (1997). Modeling Piezoelectric Stack Actuators for Control of Micromanipulation. IEEE Control Systems Magazine, vol. 17, No. 3, (June 1997), pp. 69-79.

Hu, H. \& Mrad, R. B. (2002). On the Classical Preisach model for hysteresis in piezoceramic actuators. Mechatron, vol. 13, No. 2, (March 2002), pp. 85-94.

Hughes, D. \& Wen. J. T. (1995). Preisach Modeling of Piezoceramic and Shape Memory Alloy Hysteresis, $4^{\text {th }}$ IEEE Conf. on Control Applications, pp. 1086-1091, New York, USA, Sep., 1995.

Hwang, C. L. \& Jan, C. (2003). A Reinforcement Discrete Neuro-Adaptive Control for Unknown Piezoelectric Actuator Systems with Dominant Hysteresis. IEEE Transactions on Neural Networks, vol. 14, No. 1, (Jan 2003) pp. 66-78.

Janocha, H. \& Kuhnen, K. (2000). Real-time Compensation of Hysteresis and Creep in Piezoelectric Actuators. Sensors \& Actuators A: Physical, vol. 79, No. 2, (Feb. 2000), pp. 83-89.

Kuhnen, K. \& Janocha, H. (2001). Inverse Feedforward Controller for Complex Hysteretic Nonlinearities in Smart-Material Systems. Control and Intelligent Systems, vol. 29, (2001), pp. 74-83.

Kuhnen, K. \& Janocha, H. (2002). Complex hysteresis modeling of a broad class of hysteretic nonlinearities, $8^{\text {th }}$ Int. Conf. on New Actuators, Bremen, June 2002.

Landauer, R.; Young, D. R. \& Drougard, M. E. (1956). Polarization reversal in the barium titanate hystersis loop. Journal of Applied Physics, vol. 27, No. 71, (1956) pp 752-758.

Riviere, C. N.; Ang, W. T. \& Khosla, P. K. (2003). Toward Active Tremor Canceling in Handheld Microsurgical Instruments. IEEE Transactions on Robotics and Automation, vol. 19, No. 5, (Oct. 2003), pp. 793-800.

Stepanenko, Y. and Su, C. Y. (1998). Intelligent Control of Piezoelectric Actuators, IEEE Conf. on Decision \& Control, pp. 4234-4239, Tampa, Florida USA, Dec. 1998.

Tao, G. (1995). Adaptive Control of Plants with Unknown Hystereses. IEEE Transactions on Automatic Control, vol. 40, No. 2, (Feb. 1995), pp. 200-212. 


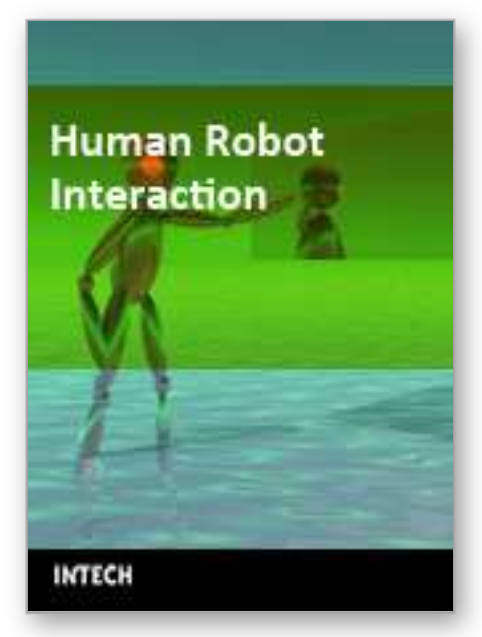

\author{
Human Robot Interaction \\ Edited by Nilanjan Sarkar
}

ISBN 978-3-902613-13-4

Hard cover, 522 pages

Publisher I-Tech Education and Publishing

Published online 01, September, 2007

Published in print edition September, 2007

Human-robot interaction research is diverse and covers a wide range of topics. All aspects of human factors and robotics are within the purview of HRI research so far as they provide insight into how to improve our understanding in developing effective tools, protocols, and systems to enhance HRI. For example, a significant research effort is being devoted to designing human-robot interface that makes it easier for the people to interact with robots. $\mathrm{HRI}$ is an extremely active research field where new and important work is being published at a fast pace. It is neither possible nor is it our intention to cover every important work in this important research field in one volume. However, we believe that $\mathrm{HRI}$ as a research field has matured enough to merit a compilation of the outstanding work in the field in the form of a book. This book, which presents outstanding work from the leading $\mathrm{HRI}$ researchers covering a wide spectrum of topics, is an effort to capture and present some of the important contributions in $\mathrm{HRI}$ in one volume. We hope that this book will benefit both experts and novice and provide a thorough understanding of the exciting field of HRI.

\title{
How to reference
}

In order to correctly reference this scholarly work, feel free to copy and paste the following:

U-Xuan Tan, Win Tun Latt, Cheng Yap Shee, Cameron Riviere and Wei Tech Ang (2007). Modeling and Control of Piezoelectric Actuators for Active Physiological Tremor Compensation, Human Robot Interaction, Nilanjan Sarkar (Ed.), ISBN: 978-3-902613-13-4, InTech, Available from:

http://www.intechopen.com/books/human_robot_interaction/modeling_and_control_of_piezoelectric_actuators _for_active_physiological_tremor_compensation

\section{INTECH}

open science | open minds

\author{
InTech Europe \\ University Campus STeP Ri \\ Slavka Krautzeka 83/A \\ 51000 Rijeka, Croatia \\ Phone: +385 (51) 770447 \\ Fax: +385 (51) 686166 \\ www.intechopen.com
}

\author{
InTech China \\ Unit 405, Office Block, Hotel Equatorial Shanghai \\ No.65, Yan An Road (West), Shanghai, 200040, China \\ 中国上海市延安西路65号上海国际贵都大饭店办公楼 405 单元 \\ Phone: +86-21-62489820 \\ Fax: +86-21-62489821
}


(C) 2007 The Author(s). Licensee IntechOpen. This chapter is distributed under the terms of the Creative Commons Attribution-NonCommercial-ShareAlike-3.0 License, which permits use, distribution and reproduction for non-commercial purposes, provided the original is properly cited and derivative works building on this content are distributed under the same license. 